\title{
Agronegocios
}

\section{Pre-factibilidad para una plantación de una hectárea de maracuyá en San Pablo de Turrubares.} Pre-feasibility study for the establishment of a hectare of passion
fruit in San Pablo of Turrubares.

Jorge Flores-Peralta', David Barboza-Navarro², Nataly Montero-Solís Carlos $^{3}$, González-Blanco ${ }^{4}$

Flores Peralta, J., Barboza Navarro, D., Montero Solís, N., \& González Blanco, C. (2019). Prefactibilidad para una plantación de una hectárea de maracuyá en San Pablo de Turrubares. Revista E-Agronegocios, 5(1). https://doi.org/10.18845/rea.v5i1.4030

Vol. 5. №1. Enero-junio, 2019 DOI: https://doi.org/10.18845/rea.v5i1.4030

\footnotetext{
${ }^{1}$ Estudiante de Economía Agrícola y Agronegocios. Universidad de Costa Rica. Costa Rica. Correo electrónico: arturoflores777@gmail.com.

${ }^{2}$ Estudiante de Economía Agrícola y Agronegocios. Universidad de Costa Rica. Costa Rica.

${ }^{3}$ Estudiante de Economía Agrícola y Agronegocios. Universidad de Costa Rica. Costa Rica.

${ }^{4}$ Estudiante de Economía Agrícola y Agronegocios. Universidad de Costa Rica. Costa Rica.
} 


\section{Resumen}

Con este estudio de prefactibilidad se pretende conocer la viabilidad económica de un sistema de producción de maracuyá amarillo en la zona de San Pablo de Turrubares, en la provincia de San José, que cuenta con las condiciones edafoclimáticas óptimas para el desarrollo y la producción de la planta, considerando la comercialización del fruto en el mercado mayorista en el Centro Nacional de Abastecimiento y Distribución de Alimentos (CENADA).

También se pretende generar conocimiento acerca de un producto no tradicional en el sector primario de la economía costarricense, fomentando el desarrollo, siendo esto una herramienta de consulta para productores interesados en conocer acerca de la rentabilidad del cultivo en cuanto a oportunidades de comercialización, y así fomentar la diversificación agrícola nacional a través de los productos no tradicionales.

El periodo de evaluación para el proyecto fue de 7 años, tiempo que abarca dos ciclos completos del cultivo, lo que permitió conocer si el mismo es rentable a un mediano plazo. Para esto se elaboró y desarrolló el estudio técnico, socioeconómico y político, organizacional y legal, financiero y de mercado y un análisis de aspectos ambientales. Al finalizar el periodo de evaluación se obtuvo un Valor Actual Neto (VAN) de 1.073 .470 colones y una Tasa Interna Retorno (TIR) del 20,42\%, lo anterior a una tasa de costo de capital del $19,05 \%$.

Palabras clave: Cultivo de maracuyá, preparación y evaluación de proyectos, prefactibilidad de proyectos.

\section{Abstract}

With this pre-feasibility study, the aim is to know the economic viability of a passion fruit production system in the San Pablo de Turrubares area, which has the optimum soil and climate conditions for the development and production of this crop, considering the commercialization of the fruit in the wholesale market CENADA.

It also aims to generate knowledge about a non-traditional product in the primary sector of the Costa Rican economy, promoting rural development. This is positive because interested producers can study this report to learn about the profitability of the crop in terms of marketing opportunities to encourage national agricultural diversification through non-traditional products.

The evaluation period for the project is 7 years because by this date two complete cycles of the crop will have been obtained, which will allow knowing in the medium term if it is profitable or not.

In addition, the costs in which the organization must incur, payment for tax and labor obligations, investments and necessary reinvestments, as well as the income that the activity generates are presented. For this, technical, socioeconomic and political, organizational and legal, financial and market studies are developed. At the end of the

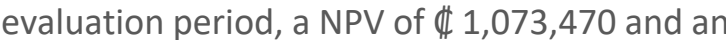
IRR of $20.42 \%$ were obtained, at a capital cost rate of $19.05 \%$.

Key words: Passion fruit crop, preparation and evaluation projects, prefeasibility projects 


\section{Introducción}

El maracuyá pertenece a la familia de las pasifloras (passifloraceae), que cuenta con alrededor de 630 especies, de las cuales, el género más importante es Passiflora. Esta engloba cerca de 530 especies que se encuentran distribuidas a lo largo del continente americano (Miranda et al., 2009). Barquero (2017) menciona que existen ocho productos agrícolas con alto potencial exportador, entre ellos el maracuyá, los cuales pueden producirse en mayor medida en Costa Rica para ser comercializados en el mercado internacional.

Este estudio de prefactibilidad se realizó con el fin de conocer la rentabilidad de la producción de una hectárea (ha) de maracuyá en el cantón de Turrubares en la provincia de San José, debido a que actualmente, el sector agropecuario nacional puede presentar problemas relacionados con la competencia desleal, causando que los pequeños productores pierdan competitividad en el mercado nacional e internacional (Córdoba, 2011).

Por último, el proyecto se llevó a cabo en dicha localidad por recomendación del experto Carlos Saborío, debido a que la zona cuenta con las mejores condiciones edafoclimáticas para dicha actividad lo cual generará los mayores rendimientos por ha.

\section{Referente teórico}

Pardo (2004) define un estudio de prefactibilidad como una etapa de la preparación y ejecución de un proyecto de inversión, que se realiza posterior a la formulación de la idea inicial, y anterior al estudio de factibilidad, en el cual, se detallan los aspectos como términos de referencia, profundizando en los cálculos, estimaciones e investigaciones, a fin de identificar las alternativas convenientes, que se estudiarán como factibles.

El fruto del maracuyá tiene un peso promedio de 100 a 150 gramos por unidad, y se empacan en Costa Rica en cajas de cartón alrededor de 33 frutas para un contenido de $3 \mathrm{~kg}$ (Aguilar, 2013). El precio no es constante debido a que, durante ciertos períodos, existe escasez en la producción del cultivo, y en otros, una sobreproducción. CasaLuker (2010) agrega que este producto se comporta como un commodity y producto de temporada, por lo que su precio es cíclico.

Actualmente, en el mundo, más de 40 países cultivan maracuyá con fines comerciales, y su producción ha venido en aumento desde la década de los años 80 . Brasil es el principal productor a nivel mundial ya que, por sus condiciones edafoclimáticas, cosecha maracuyá casi todo el año. Seguidamente se encuentran Ecuador, Colombia, Perú con gran potencial productivo, y en menor medida a Costa Rica (Ayala y Cervallos, 2013). Según Ayala y Cervallos (2013), los principales países importadores de esta fruta a nivel mundial son Estados Unidos, Holanda y Japón. Sin embargo, en Trade Map (1999-2018) se visualizan otros países del continente asiático como China, que han tenido un crecimiento importante en cuanto a la demanda de maracuyá.

La Organización para la Cooperación y el Desarrollo Económico (OCDE) menciona que, si Costa Rica desea mantener una participación competitiva en el sector agroeconómico, debe acelerar los procesos de diversificación de cultivos, en especial los no tradicionales, y generar valor agregado a los productos obtenidos. El Ministro de Agricultura del periodo 2014-2018, Luis Felipe Arauz, mencionó que es necesario impulsar la inclusión de productos no tradicionales en los sistemas productivos del ámbito nacional (Rodríguez, 2017). 


\section{Metodología}

Para la elaboración del presente estudio de prefactibilidad se realizó una búsqueda de información en fuentes de información primaria y secundaria, como referencias bibliográficas y entrevistas a expertos, para así generar conocimiento sobre la actividad productiva del maracuyá, así como el contexto mundial y nacional que tiene el cultivo, en el sector económico, social, político y ambiental.

La recopilación de información por medio de fuentes primarias se basó en entrevistas a expertos sobre el cultivo, para conocer de primera mano los aspectos técnicos del mismo, así como legales y organizacionales que fortalecieran la base del estudio. Se consultaron también bases de datos como el Programa Integral de Mercadeo Agropecuario (PIMA) y Trade Map, para conocer los precios históricos y volúmenes del cultivo tanto a nivel nacional como mundial.

Para la proyección del precio, se tomaron datos históricos y que se convirtieron en precios reales para obtener el precio exacto en el cual se iguala la oferta y la demanda sin efecto de la inflación, luego se obtuvo un promedio sobre los mismos para aplicar la inflación proyectada por el Banco Central de Costa Rica (BCCR) para los años que abarca el estudio. Además, para la proyección de volúmenes se tomaron los datos semanales obtenidos por el PIMA.

En el estudio de aspectos ambientales se elaboró una matriz de Leopold para conocer los aspectos ambientales que afectan el proyecto. En lo referente al estudio organizacional y legal, se consultó sobre todas las obligaciones legales con las cuales el proyecto debe cumplir, para este proceso se tomó la decisión de inscribir la empresa como una sociedad anónima.

Por último, se realiza un flujo de efectivo para conocer la rentabilidad final del proyecto, utilizando el análisis Monte Carlo en la sensibilización del flujo, el cual implica definir las variables que permiten determinar la distribución de probabilidad para el parámetro dado del proyecto, y así conocer que tan sensibles son las variables escogidas a cambios en ellas.

\section{Resultados y discusión}

\section{Entorno económico, político y social del proyecto}

Según (INEC, 2014), se estima que Turrubares cuenta una población total de 6.703 habitantes para el año 2018, con una densidad promedio de 16,14 personas por $\mathrm{km}^{2}$ de acuerdo a su superficie total. PEN e INEC (2013) determinan que, para el 2011, un 13,4\% de la población vivía en zonas urbanas, mientras que el $86,6 \%$ de pobladores lo hacía en zonas rurales, mostrando un incremento de $2,85 \%$ en el caso de zonas urbanas, respecto al año 2000. En el caso de la población nacida en el extranjero, esta representa un $4,5 \%$ del total de la población del cantón, y solamente el 18,1\% de la población no se encuentra asegurada.

Tal y como se menciona a continuación, el cantón de Turrubares depende en primera instancia de las actividades del sector terciario de la economía; sin embargo, las fuentes de ingreso generadas por actividades agrícolas son de suma importancia para el desarrollo económico y social. Del total de personas catalogados como "fuerza de trabajo", el 32,9\% se dedicaba a laborar en el sector primario de la economía, el cual es conformado por el $40 \%$ del total de hombres. Por otra parte, el $13 \%$ de la población total del cantón se dedicaba al sector secundario y un $54 \%$ al terciario.

\section{Organización y factores políticos}


En la última evaluación de la Contraloría de la República sobre el Índice de Gestión Municipal (IGM) del período 2016, la Municipalidad de Turrubares fue la peor evaluada de los 81 cantones que abarcaron en el estudio, ya que tuvo una calificación menor a 27 puntos de 100 posibles, esto tiene incidencia sobre el Índice de Bienestar Material (IBM) ya que Turrubares ocupa el puesto 57 de la escala de 81 cantones, con una calificación de 0,446 (PNUD y UCR, 2011), y sobre el Índice de Progreso Social (IPS), donde tiene una calificación aproximada de 73,03, en escala de 100, ubicándolo como el cantón número 49 de la lista de los 82 cantones de Costa Rica.

Finalmente, PNUD y UCR (2011) determinaron que el Índice de Desarrollo Cantonal para Turrubares es de 0,805, ubicándose en la posición 20 de los 81 cantones, lista que encabeza el cantón de Santa Ana; además, posee una esperanza de vida de 85 años, la más alta de sus últimos años evaluados.

\section{Estudio de mercado}

\section{Descripción del mercado internacional de maracuyá}

León (2013) menciona que desde la década de los años 90 la Unión Europea, seguida por Estados Unidos, Canadá, Venezuela, México, Jamaica y República Dominicana, han sido un mercado potencial para la exportación de maracuyá. Además, Aguilar (2013) menciona que para el 2010 Brasil producía cerca de 450.000 toneladas (ton) anuales, seguido de Ecuador con 85.000, Colombia con 75.000, China con 19.000 y Perú con 15.000 ton respectivamente; mientras que en Costa Rica la actividad no era muy desarrollada.

Para la subpartida arancelaria 0810.90, que contempla frutos como anacardo, jaca, tamarindo, litchi, sapolillo, maracuyá, carambola, entre otros, se encuentran tres países que han sido constantes compradores, estos son Nicaragua, El Salvador y Honduras, siendo El Salvador el mayor importador desde el 2002 hasta el 2017 transando aproximadamente 7.610 ton de frutas; Honduras es el país que se encuentra en segunda posición con 6.731 ton importadas.

En lo que respecta a las importaciones nacionales, Nicaragua representa la mayoría de las importaciones nacionales. Desde el año 2001, la cantidad importada se ha mantenido entre las 600 y 1.000 toneladas, teniendo un pico bajo en el año 2011 y la mayor cantidad importada en el año 2013.

\section{Descripción del mercado nacional de maracuyá}

Datos de INEC (2015), muestran que para el 2014 existían cerca de 284 fincas que dedicaban parte de su extensión a la producción de maracuyá, las cuales aportaban cerca de 150,7 ha cultivadas, con un $87,33 \%$ de ha en edad de producción. Según Ulloa (2017) y Saborío y Loría (2012), se estima que actualmente, el área total del territorio nacional destinada a la siembra y producción de maracuyá es de 300-320 ha, distribuidas principalmente en las regiones Huetar Norte y Caribe, mismas que no poseen el clima más apto para la maximización de la producción en $\mathrm{t} \mathrm{ha}^{-1}$, donde la provincia de Alajuela es la que cuenta con mayor cantidad de ha sembradas y en producción, seguido por Cartago y Limón (Figura 1). 


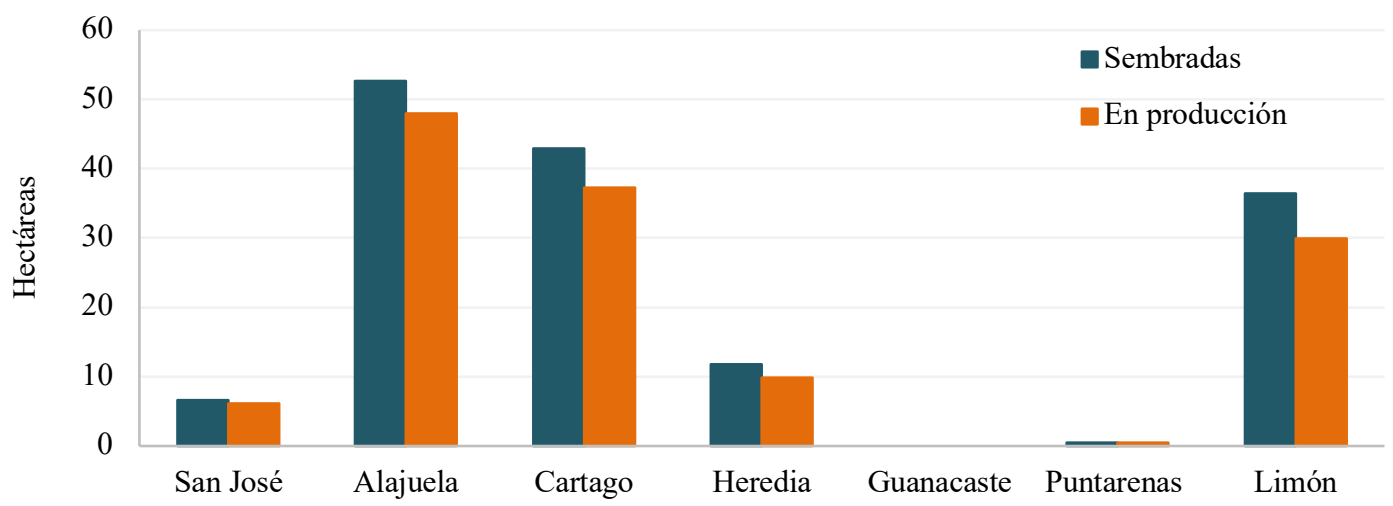

Figura 1. Costa Rica: Hectáreas sembradas y en producción de maracuyá según provincia, 2014 Fuente: Elaboración propia con base en INEC (2015).

Además, la mayor cantidad de fincas destinaban la producción de su cultivo al autoconsumo, con el $35,56 \%$ del total de fincas censadas. El segundo destino con mayor importancia es el mercado local, con un $29,23 \%$, debido a que el producto se destina a sodas, restaurantes y hoteles que ofrecen productos frescos, postres y refrescos a base de maracuyá (Figura 2).

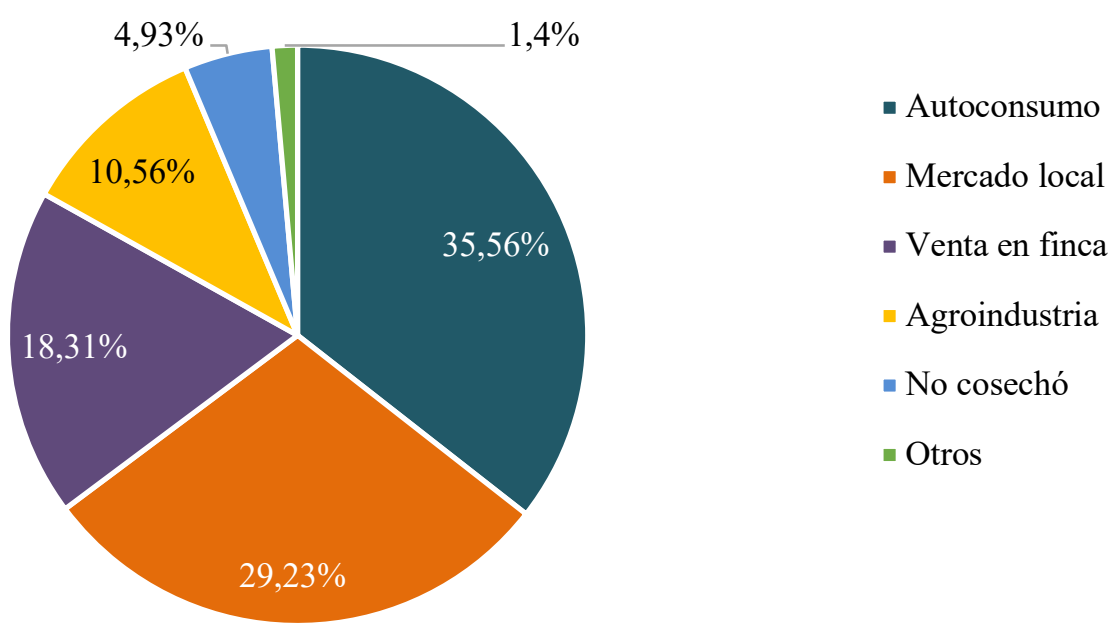

Figura 2. Costa Rica: Distribución porcentual de los destinos de la producción de maracuyá, 2014

Fuente: Elaboración propia con base en INEC (2015).

\section{Caracterización de oferta, demanda y precios para el proyecto}

A nivel nacional, uno de los principales mercados para la comercialización de maracuyá como fruta fresca es el CENADA. Por lo tanto, se partirá del supuesto de que este mercado logra un punto de equilibrio entre la oferta y la demanda a un precio determinado, asumiendo que en Costa Rica la oferta de este producto es igual a la demanda como fruta fresca.

Se procedió a calcular una función para estimar los volúmenes anuales que se transarán en CENADA durante el periodo de evaluación del proyecto a partir de los datos de enero del 2000 hasta diciembre 2017, y una estimación de los datos de 2018 según el comportamiento hasta abril del mismo año, obteniendo como resultado 309.720 kg para 2019 y un crecimiento de 13.362 kg para 
cada año siguiente (Figura 3). Además, se calcularon límites superiores e inferiores para las proyecciones a \pm 1 desviación estándar.

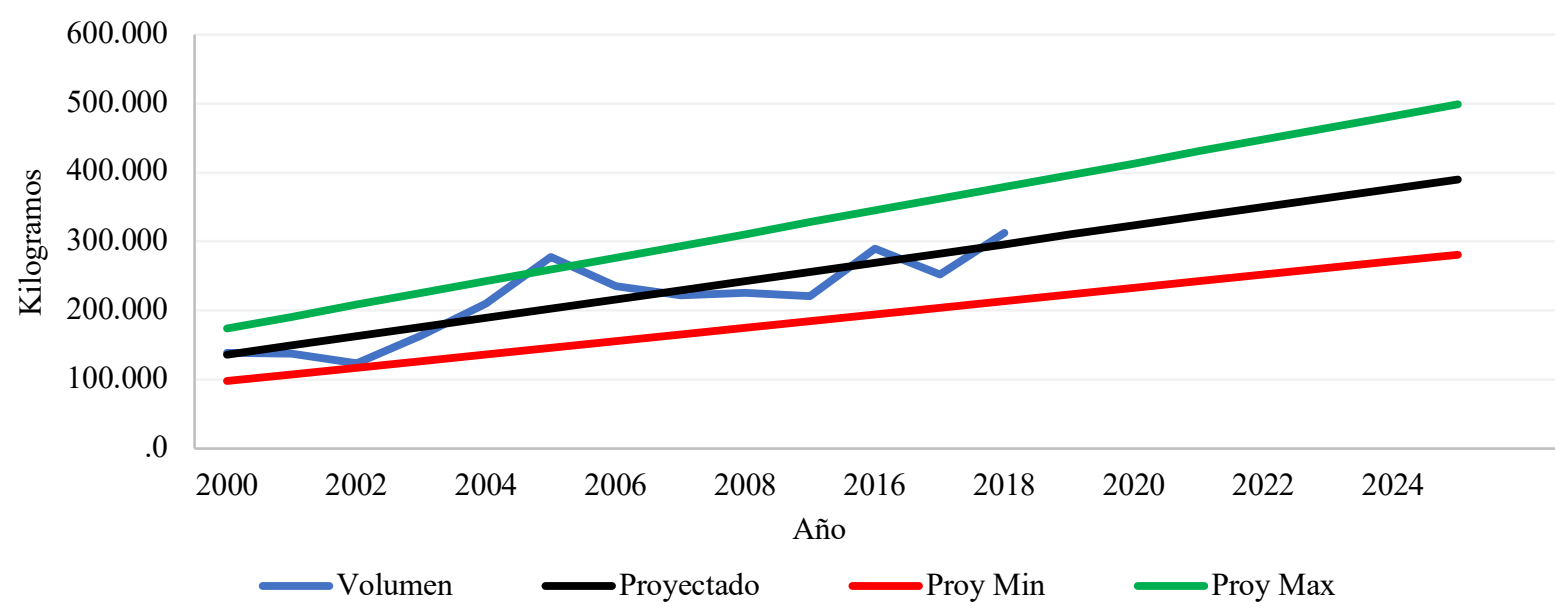

Figura 3. Costa Rica: Volúmenes históricos y proyecciones en kilogramos de maracuyá transados por año en el CENADA a \pm 1 desviación estándar, 2000-2025

Fuente: Elaboración propia con base en PIMA (2018).

Para estimar un volumen aproximado de la cantidad de maracuyá transada en CENADA, se procedió a eliminar los periodos de crecimiento con el fin de mantener únicamente los datos cuando el mercado es estable, sin efecto de alguna externalidad debido a que se consideran como una anormalidad en la tasa de crecimiento del mercado (Figura 4). Al calcularse el promedio, se obtiene una cantidad de $1.573 \mathrm{~kg}$ con una desviación estándar de 315,38 kg.

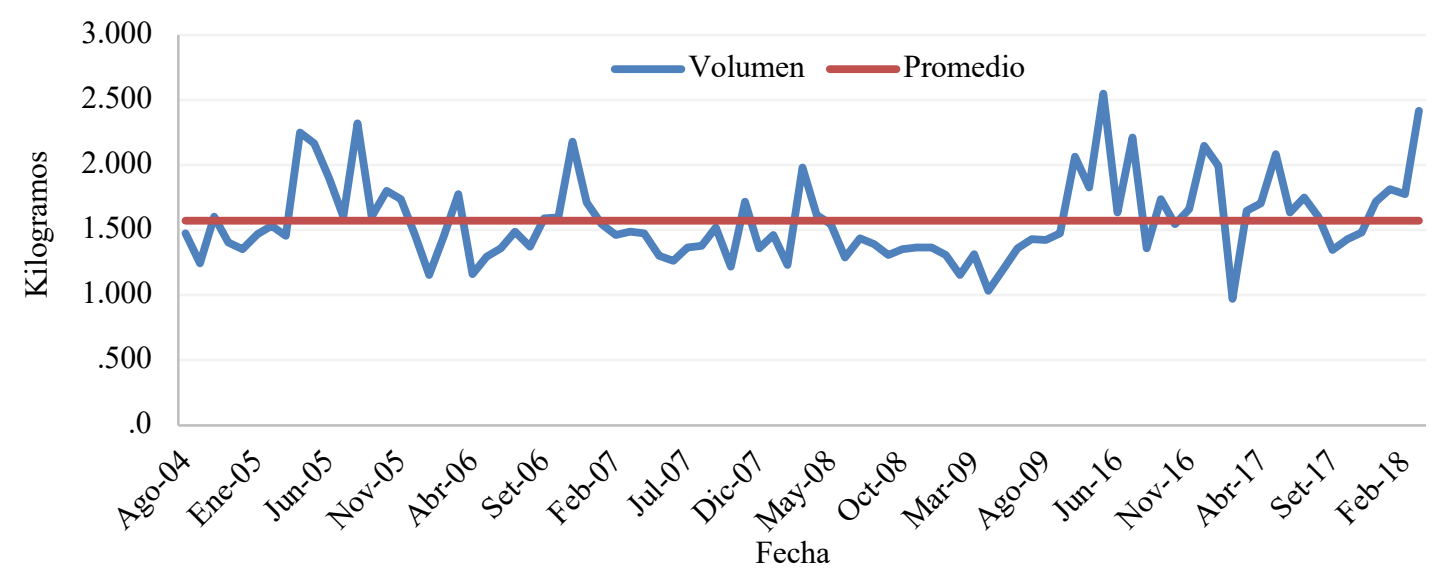

Figura 4. Costa Rica: Promedio de kilogramos de maracuyá transados por plaza en el CENADA, desde agosto 2004 a noviembre 2009 y mayo 2016 a marzo 2018

Fuente: Elaboración propia con base en PIMA (2018).

Para un mejor análisis y proyección de precios, se convirtieron los precios nominales a reales y se calculó un promedio de los precios desde enero-2001 a abril-2018, esto con el fin de determinar el precio en el que la oferta iguala a la demanda sin efecto de la inflación, el cual presenta un valor de 808 colones por kilogramo de maracuyá con una desviación estándar de 105,69 colones, siendo este precio el mejor estimador del precio real futuro. 
Debido a que las ventas se deben pronosticar con precios nominales, se procedió a agregar el efecto de la inflación proyectada por el BCCR para los precios de maracuyá amarillo en el periodo de estudio. La entidad bancaria proyecta una inflación entre el $2-4 \%$, por lo que se procedió a incrementar los precios un 3\% para cada año, siendo la proyección promedio para el 2019 de 839 colones/kg y de 1.002 colones/kg $\pm 13,10 \%$ para 2025 (Figura 5).

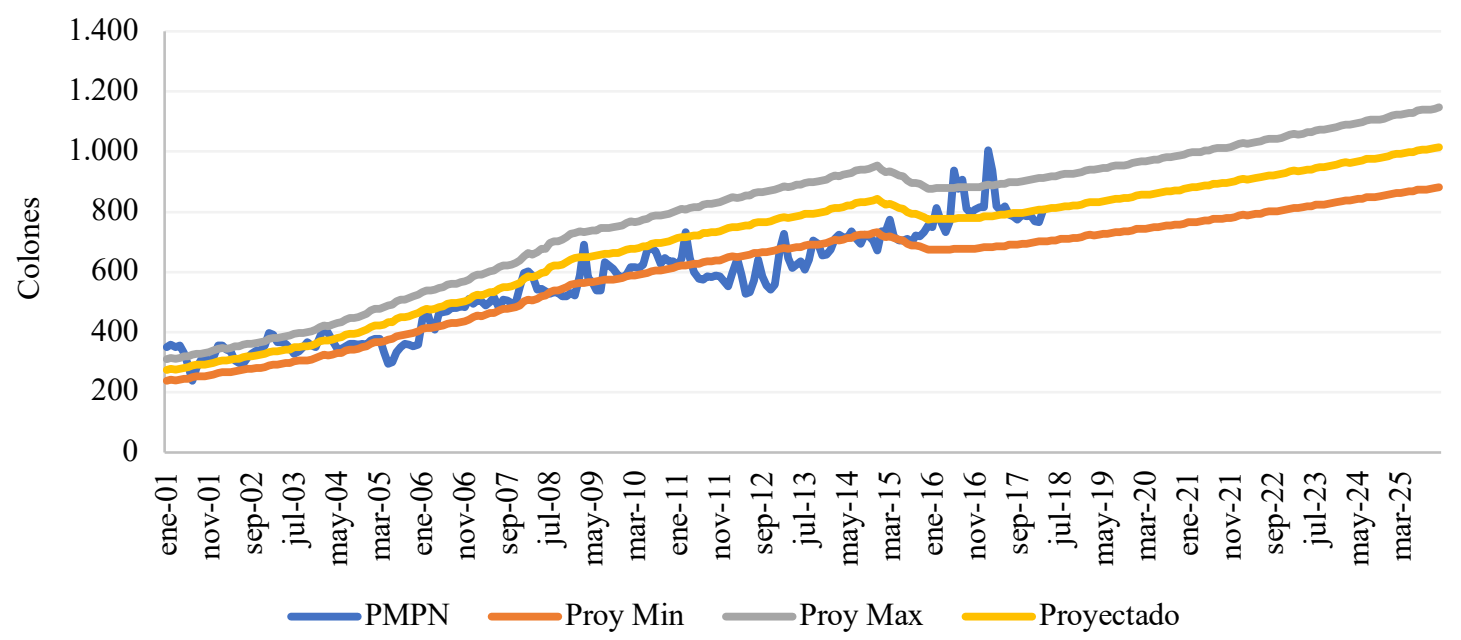

Figura 5. Costa Rica: Proyección del precio nominal promedio por kilogramo de maracuyá por plaza en el CENADA, 2001-2025

Notas: "PMPN" referencia el Promedio Mensual de Precio Nominal; "Proy Min" la Proyección Mínima; y, "Proy Max" la Proyección Máxima

Fuente: Elaboración propia con base en PIMA (2018).

Después de un análisis detallado para establecer cómo se llevará a cabo la comercialización del fruto fresco de maracuyá en el mercado de CENADA, se determinó que, por el efecto de los costos de comercialización y la especialización de mercado, el método más óptimo es el "pago por comisión" a un comercializador de la zona de Turrubares que asista a todas las plazas semanales en las ferias mayoristas. Este comercializador aprovechará su posicionamiento en el mercado para comercializar el maracuyá, y a cambio recibirá el ingreso por ventas del $20 \%$ de la producción, absolviendo al proyecto de incurrir en gastos de transporte y comercialización del mismo.

\section{Estudio técnico}

Es el aspecto más importante a considerar para establecer un sistema productivo de maracuyá recae en las condiciones edafoclimáticas, siendo necesarias 11 horas de luz diarias, una altitud de 400 a $1000 \mathrm{msnm}$, con humedad del $60 \%$ y una temperatura de $21^{\circ} \mathrm{Co} 32^{\circ} \mathrm{C}$. La precipitación debe ser de 800 a $1750 \mathrm{~mm}$ al año, con una profundidad de suelo mayor a $50 \mathrm{~cm}$ y con buen contenido de materia orgánica [Saborío y Loría, (2012), Burbano y Villanueva (2010)]. Una vez que se prepara el terreno se establece una distribución de siembra de $2 \mathrm{~m}$ entre hileras y $2,5 \mathrm{~m}$ entre plantas, para un total de 2000 plantas ha ${ }^{-1}$. Se debe colocar una espaldera con dos hilos de alambre, construida con postes de 2,5 $\mathrm{m}$ de altura, los cuales deben ser plantados a $60 \mathrm{~cm}$ según (Burbano y Villanueva, 2010) y a $50 \mathrm{~cm}$ de profundidad según Saborío y Loría (2012), distribuidos a $5 \mathrm{~m}$ uno del otro.

Según la densidad de 2.000 plantas ha-1 ${ }^{-1}$ se estima un rendimiento por hectárea de $26 \mathrm{t}$ para el primer año de producción y 52 t para el segundo, y a partir del tercer año presenta una producción 
de $13 \mathrm{~kg} \mathrm{planta}^{-1}$ anuales. Sin embargo, para este proyecto se estimarán rendimientos anuales $23,1 \%$ menores a los mencionados, con el fin de equiparar los rendimientos ofrecidos y promedio nacional, pero ligeramente más altos debido a que la mayoría de la producción en Costa Rica no se ubica en lugares aptos para el cultivo ni reciben las prácticas agrícolas que exploten el potencial genético de la planta (Saborío, 2018).

\section{Estudio financiero y análisis de sensibilidad}

En este apartado se analizan los costos e ingresos asociados a las actividades mencionadas en los apartados de estudio de mercado, organizacional-legal, técnico, y de aspectos ambientales a un plazo de 7 años. El plazo definió de esta manera debido a que el maracuyá tarda 7 meses desde la siembra hasta dar la primera cosecha, y su periodo de cosecha se extiende durante 36 meses más, por lo que durante este horizonte de tiempo se realizarían dos siembras, perdiéndose únicamente los últimos tres meses de la última cosecha.

\section{Inversiones y costos en activos fijos y alquiler de terreno y aspectos legales}

Para este rubro se toman los datos mencionados en el estudio técnico abordando el costo de alquiler de la hectárea de terreno en San Pablo de Turrubares y la construcción del galerón para el almacenamiento de insumos agropecuarios y equipo, siendo el costo de alquiler para el año 1 de 1.400.000 colones y un aumento anual del $4 \%$ y el costo total de la construcción del galerón 2.841.111 colones, calculado mediante las tipologías de MH (2015).

Se determinó que el proyecto funcionará bajo una sociedad anónima ante el Sistema Jurídico y Tributario de Costa Rica, por lo que se debe incurrir a una serie de inversiones para operar bajo la legalidad, las cuales ascienden a 243.713 colones aproximadamente.

\section{Inversión en capital de trabajo}

Según Sapag y Sapag (2008), los principales métodos para calcular el monto de la inversión en capital de trabajo son el contable, el periodo de desfase y el déficit del acumulado máximo; siendo el periodo de desfase, el cual consiste en determinar la cuantía de los costos de operación que debe financiarse desde el momento en que se efectúa el primer pago por la adquisición de la materia prima hasta el momento en que se recauda el ingreso por la venta de los productos, siguiendo la fórmula a continuación:

$$
\begin{gathered}
\text { ICT }=\frac{\text { Costo operacional anual }}{365 \text { días }} * \text { Número de días de desface } \\
\text { ICT }=\frac{8.692 .625}{365} * 210
\end{gathered}
$$

Por lo tanto, el requerimiento de inversión en capital de trabajo es de 5.001.237 colones, para el año 1, aplicando la misma fórmula para los siguientes años, a lo cual se le resta el acumulado de las inversiones de capital de trabajo de años anteriores.

\section{Ingresos generados}

Los ingresos del proyecto son calculados a partir de las estimaciones de precios elaborados en el estudio de mercado, los $\mathrm{kg} \mathrm{ha}^{-1}$ son datos abordados en el estudio técnico, siendo el porcentaje de outsourcing el determinado en el estudio de mercado. Los principales crecimientos en las ventas 
totales se deben al crecimiento de los precios a una tasa del 3\%, dato basado en las proyecciones macroeconómicas del Banco Central de Costa Rica en cuanto a la inflación y al aumento de la producción cuando el cultivo.

\section{Volúmenes de maracuyá que se cubren en CENADA}

Según los datos analizados de los volúmenes tranzados en CENADA, anualmente se comercializan $45.388 \mathrm{~kg}$ de maracuyá fresca, por lo que se estima que el proyecto abarcará los siguientes porcentajes del mercado según el año:

Cuadro 4. Turrubares: Porcentaje del mercado en CENADA que el proyecto abarcará según las proyecciones

\begin{tabular}{llllllll}
\hline Rubro & Año 1 & Año 2 & Año 3 & Año 4 & Año 5 & Año 6 & Año 7 \\
Porcentaje & $2,72 \%$ & $9,24 \%$ & $13,04 \%$ & $7,61 \%$ & $4,89 \%$ & $11,41 \%$ & $13,04 \%$
\end{tabular}

Depreciaciones y valor de desecho de activos

El valor de desecho de los activos se estimó por el método contable (Sapag y Sapag, 2012), el cual es la diferencia entre el valor del activo cuando fue comprado y la depreciación acumulada del mismo hasta la fecha.

Para determinar el valor de desecho total del proyecto, se consideró el valor de desecho de los activos y el monto invertido total para el capital de trabajo. Además, se partió del supuesto que los tres meses de cosecha que sobrepasan el periodo de evaluación no poseen valor de desecho alguno.

Cuadro 5. Turrubares: Flujo de caja puro proyectado para el proyecto a través del periodo de evaluación

\begin{tabular}{|c|c|c|c|c|c|c|c|c|c|}
\hline Rubro & Año 0 & & Año 1 & Año 2 & Año 3 & Año 4 & Año 5 & Año 6 & Año 7 \\
\hline \multicolumn{10}{|l|}{$\begin{array}{l}\text { Ingresos afectos } \\
\text { a impuestos }\end{array}$} \\
\hline Ventas & & - & 5.592 .133 & 19.583 .651 & 28.476 .932 & 17.109 .890 & 11.329 .192 & 27.227 .824 & 32.051 .038 \\
\hline \multicolumn{10}{|l|}{$\begin{array}{l}\text { Egresos afectos a } \\
\text { impuestos }\end{array}$} \\
\hline Insumos y otros & & - & 1.057 .337 & 1.099 .630 & 1.143 .615 & 1.189 .360 & 1.236 .935 & 1.286 .412 & 1.337 .868 \\
\hline Mano de obra & & - & 6.235 .289 & 10.391 .905 & 10.590 .390 & 7.873 .877 & 9.100 .165 & 11.208 .883 & 11.422 .973 \\
\hline $\begin{array}{l}\text { Alquiler del } \\
\text { terreno }\end{array}$ & & - & 1.400 .000 & 1.456 .000 & 1.514 .240 & 1.574 .810 & 1.637 .802 & 1.703 .314 & 1.771 .447 \\
\hline Depreciación & & - & 230.153 & 230.153 & 230.291 & 230.291 & 239.069 & 239.069 & 241.893 \\
\hline Total de egresos & & - & 8.922 .778 & 13.177 .688 & 13.478 .536 & 10.868 .338 & 12.213 .971 & 14.437.679 & 14.774 .182 \\
\hline
\end{tabular}




\begin{tabular}{|c|c|c|c|c|c|c|c|c|}
\hline $\begin{array}{l}\text { Utilidad antes de } \\
\text { impuestos }\end{array}$ & - & (3.330.645) & 6.405 .963 & 14.998 .396 & 6.241 .553 & (884.779) & 12.790 .145 & 17.276 .857 \\
\hline Impuestos $10 \%$ & - & - & 640.596 & 1.499 .840 & 624.155 & - & 1.279 .015 & 1.727 .686 \\
\hline Utilidad Neta & - & (3.330.645) & 5.765 .367 & 13.498 .557 & 5.617 .397 & (884.779) & 11.511 .131 & 15.549 .171 \\
\hline Depreciación & - & 230.153 & 230.153 & 230.291 & 230.291 & 239.069 & 239.069 & 241.893 \\
\hline $\begin{array}{lr}\text { Egresos } & \text { no } \\
\text { afectos } & \text { a } \\
\text { impuestos } & \end{array}$ & & & & & & & & \\
\hline Inversiones & 11.940 .990 & 13.780 & - & 3.656 & 4.913 .406 & 337.062 & - & 81.917 \\
\hline $\begin{array}{l}\text { Inversión en } \\
\text { capital de trabajo }\end{array}$ & 5.001 .237 & 2.448 .030 & 173.012 & - & - & 546.785 & 191.980 & - \\
\hline $\begin{array}{lr}\text { Beneficios } & \text { no } \\
\text { afectos } & \text { a } \\
\text { impuestos } & \end{array}$ & & & & & & & & \\
\hline Valor de desecho & - & - & - & - & - & - & - & 6.523 .964 \\
\hline Flujo de efectivo & $(16.942 .226)$ & (5.562.302) & 5.822 .508 & 13.725 .192 & 934.282 & (1.529.556) & 11.558 .220 & 22.233 .112 \\
\hline
\end{tabular}

\section{Evaluación financiera del proyecto}

En el caso del valor actual neto (VAN) y la tasa interna de retorno (TIR), se requiere del cálculo de un parámetro de comparación, el cual se utilizará la tasa de costo de capital, definida por Sapag y Sapag (2008) como aquella tasa que se utiliza para determinar el valor actual de los flujos futuros que genera un proyecto y representa la rentabilidad que se le debe exigir a la inversión por renunciar a un uso alternativo de los recursos en proyectos de riesgos similares.

$$
\mathrm{k}=\mathrm{CV}+\mathrm{TB}
$$

Donde,

$k=$ Tasa de costo de capital;

$C V=$ Coeficiente de variación de los precios reales de maracuyá tranzados en CENADA desde enero del 2000 hasta abril de 2018; y,

TBP = Tasa Básica Pasiva de Costa Rica al 18 de junio de 2018.

Se utiliza esto debido a que Paniagua (2018), sugiere que se utilice el factor que más riesgo representa en el proyecto y agregarle una tasa de "cero riesgo", siendo estas el coeficiente de variación de los precios reales de maracuyá en CENADA desde enero del 2000 hasta abril del 2018 y la tasa básica pasiva (TBP) a junio de 2018 respectivamente (fórmula aplicada a continuación). 
Cabe destacar que, debido a las características del proyecto, el método adecuado a utilizar para obtener el costo de capital es el modelo CAPM $^{5}$ (Sapag y Sapag, 2018).

$$
\mathrm{k}=13,10 \%+5,95 \%=19,05 \%
$$

Para el flujo de caja puro del proyecto, el VAN obtenido con una tasa de costo de capital de 19,05\% fue de 1.073.470 colones, concluyéndose que la rentabilidad lograda es positiva, de manera que se puede recuperar la totalidad de la inversión e incrementar el valor del capital. Además, la TIR presenta un valor del $20,42 \%$, superior por un $1,37 \%$ a la tasa de costo de capital, por lo que se concluye que el proyecto presenta un rendimiento financiero mayor al parámetro de comparación establecida por los inversionistas.

\section{Análisis de sensibilidad Monte Carlo sobre la variable "precio"}

Este análisis toma en cuenta todos los precios de venta posibles del producto, y mediante el supuesto que estos siguen una distribución normal estándar, calcula la probabilidad de que el proyecto sea rentable ante todos los precios establecidos sensibilizando el VAN. El resultado fue de un $57 \%( \pm 2,5)$, lo cual significa que el proyecto es rentable en el $57 \%$ de los escenarios de precios posibles, considerando posibles caídas abruptas y elevaciones significativas debido a shocks de oferta que el modelo de proyección de precios que el proyecto no logra pronosticar.

\section{Conclusiones}

Según el estudio técnico realizado se obtendrán rendimientos mayores al promedio nacional debido a la ubicación estratégica del proyecto, aunque estos serán menores a los proyectados por Saborío (2018) por una decisión conservadora ante volúmenes de producción, exigiendo una mayor rentabilidad al proyecto.

De acuerdo a los resultados obtenidos en la matriz de Leopold, se deberá procurar el uso eficiente de los recursos naturales que permita asegurar la generación y obtención de productos alimenticios sin comprometer el bienestar ambiental.

En términos de mercado y bajo los niveles de producción proyectados, el máximo en toneladas que se venderá en el CENADA representa aproximadamente un 13,04\% del volumen transado actualmente, y se prevé que no se generará un shock de oferta que pueda afectar los precios de venta (Paniagua, 2018).

Se considera que el proyecto es rentable debido a que el VAN es mayor a cero y la TIR es mayor a la tasa de costo de capital $(19,05 \%)$. Además, es rentable con una inflación del $4 \%$ sobre costos y del $3 \%$ en los precios de venta de maracuyá con el fin de evaluar el proyecto en un escenario desfavorable. No se considera un escenario de inversión con financiamiento por medio de deuda a largo plazo pues el apalancamiento podría representar un escenario favorable para la rentabilidad del proyecto, sin considerar el factor riego sobre el pago de la deuda. Por último, el proyecto es rentable en un $57 \%( \pm 2,5)$ de los escenarios posibles analizados.

\footnotetext{
${ }^{5}$ Modelo de Valorización de Activos de Capital.
} 


\section{Literatura citada}

Aguilar, J. (2013). Análisis de Ecuador como país exportador de maracuyá con sus oportunidades de inversión en el Ecuador. (Tesis de Ingeniería en Comercio Exterior). Universidad Espíritu Santo. 80p.

Arias, J., Ocampo, J. y Urrea, R. (2014). La polinización natural en el maracuyá como un servicio productivo y ecosistémico. Agronomía Mesoamericana. 25 (1), 73-83. Recuperado de http://www.mag.go.cr/rev_meso/v25n01_073.pdf.

Ayala, A. y Cevallos, E. (2013). Plan de exportación de concentrado de maracuyá ecuatoriano al mercado japonés. (Tesis de Ingeniería en Comercio Exterior). Universidad Politécnica Salesiana. Recuperado de https://www.dspace.ups.edu.ec/bitstream/123456789/5042/1/GT000444.pdf.

Barquero, M. (2017, 16 de junio). Ocho productos agrícolas se erigen con alto potencial exportador. La Nación. Recuperado de https://www.nacion.com/economia/agro/ocho-productos-agricolas-seerigen-con-alto-potencial-exportador/PPTTNSJXQ5BZLCSL5VXYS2K5EY/story/.

Burbano, J. y Villafuerte, F. (2010). Maracuyá: sistema productivo sustentable. (Tesis de Pregrado). Guayaquil, Ecuador, Universidad Católica Santiago de Guayaquil. Recuperado de http://www.bibliotecasdelecuador.com/Record/ir-:3317-6160.

CasaLuker. (2010). Agroindustria y mercadeo del maracuyá. Recuperado de http://www.asohofrucol.com.co/archivos/biblioteca/biblioteca_160_AGROINDUSTRIA_MERCADE O_MARACUYA.pdf.

Chuquilla, D. (2012). Estudio de factibilidad para la producción del cultivo de maracuyá en una extensión de 20 hectáreas en la provincia de Santo Domingo de Los Tsáchilas. (Tesis Ingeniería Comercial). Pontificia Universidad Católica de Ecuador. Recuperado de https://issuu.com/pucesd/docs/pdf_diego_fernando_chuquilla_carrer.

Córdoba, J. (2011). Productores en problemas: Agricultura tica pelea contra competencia desleal. Semanario Universidad. Recuperado de https://semanariouniversidad.com/pais/productores-enproblemas-agricultura-tica-pelea-contra-competencia-desleal/.

INEC (Instituto Nacional de Estadística y Censos). (2014). Proyecciones de población según provincia, cantón y distrito. San José, Costa Rica. Recuperado de http://www.inec.go.cr/poblacion/temasespeciales-de-poblacion.

INEC (Instituto Nacional de Estadística y Censos). (2015). VI Censo Nacional Agropecuario (CENAGRO). San José, Costa Rica. Recuperado de www.inec.go.cr.

León, M. (2013). Estudio de factibilidad para el cultivo de maracuyá (Passiflora edulis), En El Búa, Santo Domingo De Los Tsáchilas. (Tesis Ingeniería en Agroempresas). Universidad San Francisco de Quito. Recuperado de http://repositorio.usfq.edu.ec/bitstream/23000/3019/1/109502.pdf.

Miranda, D., Fischer, G., Carranza, C., Magnitskiy, S., Casierra, F., Piedrahíta, W. y Flórez, L. (2009). Cultivo, poscosecha y comercialización. Bogotá: Ruben's Impresores Editores. Recuperado de http://www.asohofrucol.com.co/archivos/biblioteca/biblioteca_118_cultivo_poscosechavp.pdf.

Paniagua, J. (2018, 18 de junio). Criterios técnicos para la evaluación de proyectos (entrevista personal). San José, Costa Rica, UCR.

Pardo, J. (2004). Estudio de prefactibilidad de un proyecto para la prestación de servicios de acabados para vivienda de interés social en Bogotá D. C. Bogotá: Pontificia Universidad Javeriana. Recuperado de https://www.javeriana.edu.co/biblos/tesis/ingenieria/tesis45.pdf 
PEN (Programa Estado de la Nación), INEC (Instituto Nacional de Estadística y Censos). (2013). Indicadores Cantonales: San José. San José, Costa Rica. 42 p. Recuperado de https://www.estadonacion.or.cr/otras-publicaciones-costarica/indicadores-cantonales.

PNUD (Programa de las Naciones Unidas para el Desarrollo) y UCR (Universidad de Costa Rica). (2011). Atlas Del Desarrollo Humano Cantonal De Costa Rica. San José, Costa Rica. 116 p. Recuperado http://www.cr.undp.org/content/dam/costa_rica/docs/undp_cr_atlas_cantonal.pdf.

PIMA (Programa Integral de Mercadeo Agropecuario). (2018). Información y caracterización de CENADA. San José, Costa Rica. Recuperado de http://www.pima.go.cr/quienes-somos/.

Rodríguez, A. (2017, 09 de abril). OCDE recomienda a Costa Rica acelerar sus procesos de diversificación de productos y aumentar valor agregado). El Financiero. Recuperado de https://www.elfinancierocr.com/economia-y-politica/ocde-recomienda-a-costa-rica-acelerar-susprocesos-de-diversificacion-de-productos-y-aumentarvalor/HOB32Q3W3VET7M7WIM4CU75ZGM/story/.

Saborío, C. (2018, 20 de junio). Criterio técnico para el establecimiento del cultivo de maracuyá (conversación vía telefónica). UCR, San José, Costa Rica.

Saborío, C. y Loría, C. (2012). El cultivo de maracuyá "establecimiento y desarrollo". UCR, San Jose, Costa Rica. Recuperado de https://es.scribd.com/document/211224506/Manual-de-MaracuyaCdp.

Sapag, N. y Sapag, R. (2008). Preparación y evaluación de proyectos. (5. ${ }^{a}$ ed.). Bogotá: McGraw-Hill. $463 \mathrm{p}$.

Ticolndex. (2018). Historia del cantón de Turrubares. Costa Rica. Recuperado de http://www.ticoindex.com/turrubares/datos/historia+de+turrubares.html.

Trade Map. (1999-2018). Transacciones comerciales de la subpartida 0810.90. Internacional Trade Centre. Disponible en https://trademap.org.

Ulloa, L. (2017). Oportunidad de comercialización de productos incipientes. San José: Procomer. Recuperado de https://www.procomer.com/uploads/downloads/f62761458ba1270c78d0d69f8c0d341229912033 .pdf. 